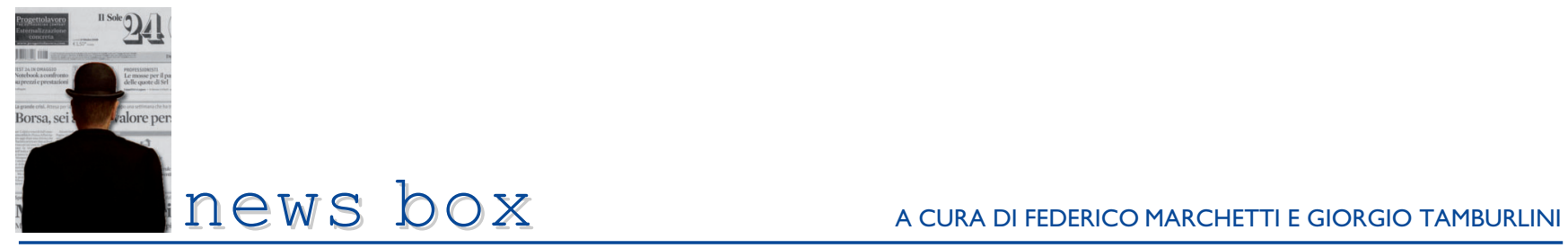

\title{
UNA BUONA PARTENZA NELLA VITA PER TUTTI
}

Per un sistema integrato di politiche, servizi e interventi , capace di garantire a tutte le bambine e a tutti i bambini le migliori opportunità di sviluppo nei primi anni di vita

Pubblichiamo una sintesi infografica del documento (che può essere scaricato da https://www.alleanzainfanzia.it/wp-content/uploads/2022/02/Sistema-integrato-0-6.pdf), prodotto da un Gruppo di lavoro multidisciplinare (educatori, psicologi, pedagogisti, pediatri, studiosi di scienze sociali) coordinato dal Centro per la Salute del Bambino onlus, che è stato fatto proprio dallintera rete di organizzazioni ed esperti che fanno parte di Alleanza per l'Infanzia.

\section{Finalità della proposta}

A PROMUOVERE lo sviluppo complessivo del bambino e un ruolo attivo delle comunità a sostegno di: benessere, sviluppo ed educazione dei bambini.

B PREVENIRE l'insorgere precoce delle diseguaglianze, della povertà educativa e del maltrattamento.

c SOSTENERE tutte le famiglie e in particolare quelle con bambini con bisogni speciali.

\section{II problema}

Come possiamo rispondere ai bisogni di sviluppo dei bambini (salute, nutrizione, educazione, protezione sociale, genitorialià responsiva) visto che sono tra loro strettamente connessi?

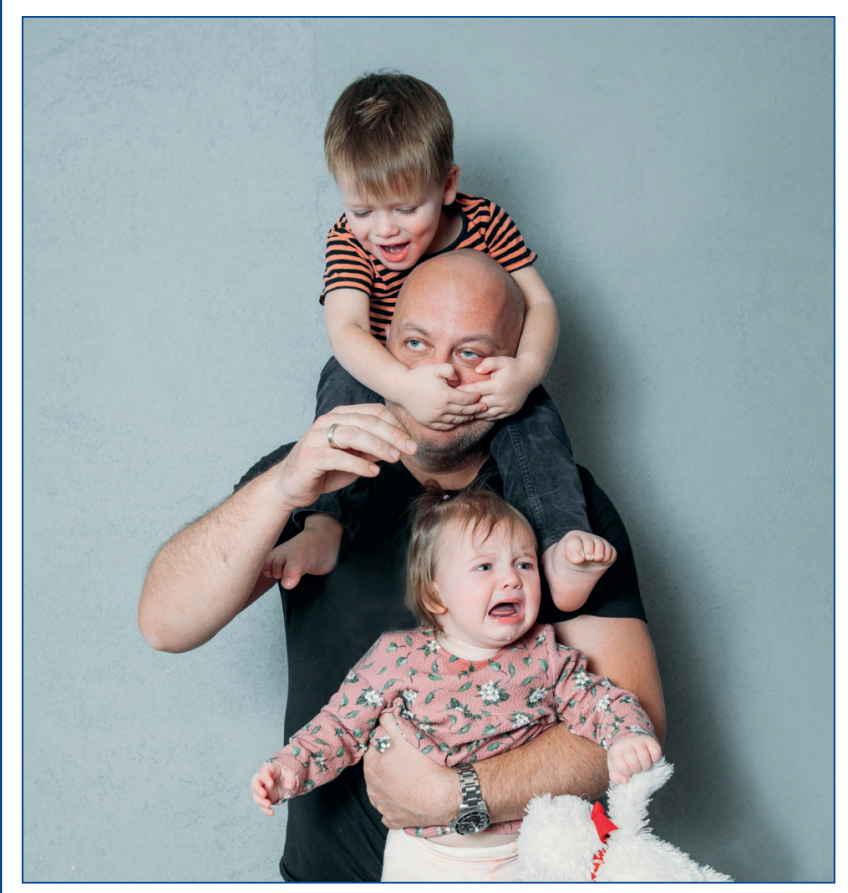

\section{Le risposte}

1 Perché sostenere LE FAMIGLIE?

$\square$ punto I: per l'importanza nei primi 1000 giorni di vita dell'ambiente più prossimo, soprattutto familiare.

$\square$ punto 2: perché fattori economici, sociali e culturali condizionano fortemente l'ambiente familiare e possono determinare precoci diseguaglianze nello sviluppo.

$\square$ punto 3: perché promuovere la genitorialità responsiva mitiga l'effetto di condizioni di partenza sfavorevoli.

$\square$ punto 4: perché investire risorse nei primi anni di vita è un dovere dell'intera comunità.

$\square$ punto 5: perché coinvolgere fin da subito (periodo prenatale e nascita) i padri porta tanti i benefici sia per il bambino che per i genitori.

$\square$ punto 6: per raggiungere tutte le famiglie e non perdere opportunità preziose di intervento precoce.

$\square$ punto 7: perché purtroppo l'accesso universale al nido non è ancora realtà e pochissimi genitori vengono sostenuti nel periodo prenatale e fino ai tre anni.

\section{Nurturing care}

Le diseguaglianze nella salute e nello sviluppo possono essere mitigate da interventi a sostegno delle risorse materiali e personali dei genitori tramite politiche abilitanti, servizi di accompagnamento alle competenze dei genitori e degli adulti di cura (come rappresentato nel grafico).

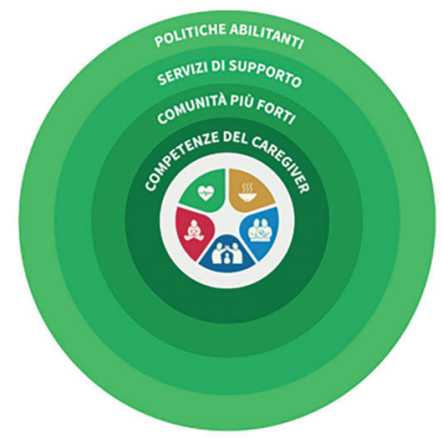




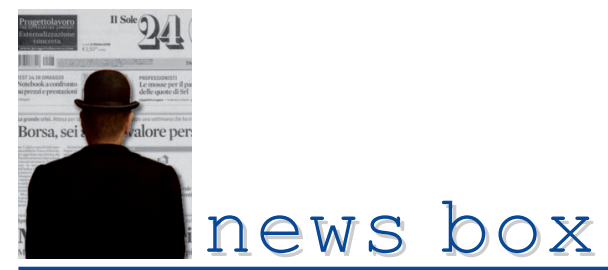

\section{Perché un approccio INTEGRATO?}

$\square$ punto I: perché il bambino è un tutt'uno e i suoi bisogni di salute, educazione, cure familiari sono strettamente interdipendenti, quindi richiedono risposte coerenti e coordinate nell'attività di promozione, prevenzione e cura, nel lavoro educativo e di sostegno per le famiglie, come indicato anche da molti documenti e linee di indirizzo a livello nazionale, non ultimo il Piano di Garanzia Infanzia.

$\square$ punto 2: perché le esperienze di formazione multiprofessionale dimostrano come percorsi formativi condivisi contribuiscano all'efficacia della rete dei servizi.

$\square$ punto 3: perché esistono esperienze locali di collaborazione tra settori che dimostrano quanto questo sia possibile e fruttuoso.

$\square$ punto 4: per favorire l'integrazione dei servizi che si occupano di infanzia e famiglie, che nella realtà italiana agiscono in modo separato e spesso non comunicante tra loro.

\section{La proposta}

1 Occorre garantire a ogni genitore di poter usufruire, in quanto livello essenziale di assistenza, di percorsi di accompagnamento nei primi periodi di vita del bambino, a partire dal periodo prenatale e per i primi due anni di vita, realizzati con il concorso di tutti i servizi (sanitari, educativi, sociali e culturali).

\section{IN PRATICA}

\section{Coordinamento}

- Meccanismo/autorità che coordini l'attività dei Ministeri competenti e ottimizzi l'uso delle risorse.

- Sostegno tecnico alla progettualità degli Enti locali nell'uso dei fondi disponibili.

- Coordinamento intersettoriale delle funzioni a livello locale per raccolta dati, elaborazione piani comuni, percorsi di formazione trasversale e interdisciplinare (figura di coordinatore).

\section{Proattività}

- Analisi delle difficoltà di accesso ai servizi.

- Approcci peer-to-peer, visite a domicilio, contatti vicini alla residenza, mediatori informali.

- Utilizzo di tutti i canali, formali e non, per raggiungere le famiglie.

- Apertura dei servizi educativi alla comunità (iniziative aperte anche a famiglie che non frequentano nido o scuola dellinfanzia).

\section{Formazione}

Gli operatori che lavorano con famiglie e bambini devono condividere momenti formativi e piani di intervento, per garantire coerenza di messaggi e facilitare l'accesso ai servizi.

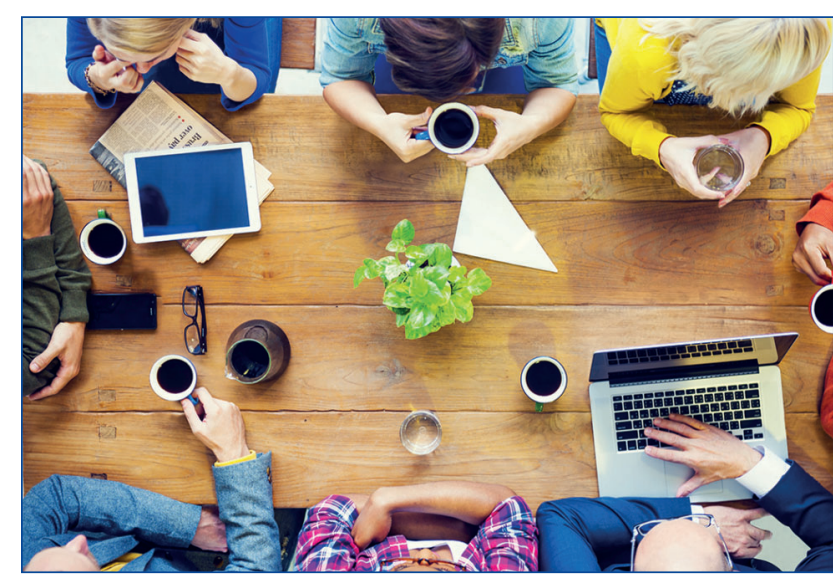

2 Occorre che i servizi per l'infanzia, e le loro articolazioni a livello territoriale, prevedano meccanismi (tavoli, conferenze permanenti, accordi di piano e di programma) di condivisione su: analisi dei bisogni, pianificazione di interventi, definizione e facilitazione di percorsi, modalità di comunicazione e di operatività proattive che consentano di raggiungere tutte le famiglie, con priorità per quelle in situazioni di vulnerabilità.

\section{IN PRATICA}

\section{Prenascita e nascita}

- Incontri su sviluppo bambino e genitorialità con entrambi i genitori prima della nascita.

- Alla nascita contatto precoce, presenza del padre, sostegno all'allattamento.

- Informazioni sui servizi offerti dalla comunità, su sostegni economici e psicosociali.

\section{0-24 mesi}

- Dialogo con il pediatra di famiglia.

- Frequenza del nido, aperto a tutte le famiglie.

- Coinvolgimento di genitori e bambini assieme in attività che sostengono sviluppo e relazione (lettura, gioco, esperienza musicale ecc.).

- Accesso a biblioteche, musei, attività culturali.

\section{Sempre}

Interventi di sostegno ulteriore per bisogni di salute, psicosociali, economici forniti dai diversi servizi.

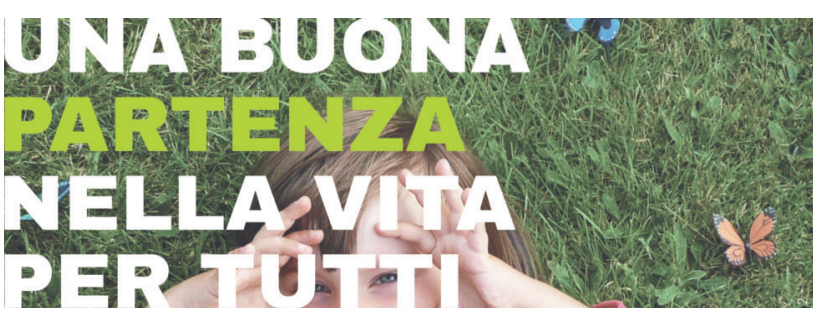

